\title{
Swarm Eye: A Distributed Autonomous Surveillance System
}

\author{
Faisal Khan ${ }^{1}$, Tarapong \\ Sreenuch $^{3}$ \\ Integrated Vehicle Health \\ Management Centre \\ Cranfield University, Bedford, UK
}

\author{
Jörn Mehnen ${ }^{2}$ \\ Manufacturing and Materials \\ Department \\ University of Strathclyde \\ Glasgow, UK
}

\author{
Syed Alam ${ }^{4, \#}$, Paul Townsend ${ }^{5, \$}$

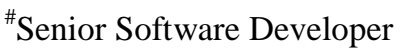 \\ Clockwork IT Limited, \\ Coventry, UK \\ ${ }^{\$}$ Lockheed Martin, UK
}

\begin{abstract}
Conventional means such as Global Positioning System (GPS) and satellite imaging are important information sources but provide only limited and static information. In tactical situations rich 3D images and dynamically self-adapting information are needed to overcome this restriction; this information should be collected where it is available. Swarms are sets of interconnected units that can be arranged and coordinated in any flexible way to execute a specific task in a distributed manner. This paper introduces Swarm Eye - a concept that provides a platform for combining the powerful techniques of swarm intelligence, emergent behaviour and computer graphics in one system. It allows the testing of new image processing concepts for a better and well informed decision making process. By using advanced collaboratively acting eye units, the system can observe, gather and process images in parallel to provide high value information. To capture visual data from an autonomous UAV unit, the unit has to be in the right position in order to get the best visual sight. The developed system also provides autonomous adoption of formations for UAVs in an autonomous and distributed manner in accordance with the tactical situation.
\end{abstract}

Keywords-Swarm intelligence; distributed surveillance system; bio-inspired algorithm; cooperative UAVs

\section{INTRODUCTION}

Nature has been a source of inspiration for today's various modern and complex technology and intriguing systems. Many inventions have been done by adopting the natural system into either mechanical or electric manner. Swarms are schools of fish, colonies of ants and termites, flocks of birds, herds of land animals. Insects such as mosquitoes, spiders and dragon flies have a compound eye which provides them with a large view angle. This helps them to quickly detect fast movements and compensate for the physical lacks of the single units by 'intelligent' combination of information, [1], [2].

A major problem with a centralized system is that if the centre of the system becomes dysfunctional the whole system may stop working. To make a system more robust, adaptable systems such as autonomous swarms look very promising [3]. The formation of pulling chains by weaver ants or the surrounding of a prey like a target by a group of predators can be seen as examples or multi-pattern formation of swarms in nature. A first engineering adoption of this idea can be seen in the swarm intelligence based multi-robot pattern formation

This article contains material from M. Res. Thesis, Cranfield University, England, UK.
[4]. This model argues that the pattern to be formed is controlled by the surroundings, and the final shape and the size are partially decided by the task or objective of each agent during the coordination.

In the approach used in [5], the focus is on collective intelligence. The rules used to organize their swarm raids has broad application for advancement in programming of multiagent autonomous systems, the design and for providing insight into the concepts of collective self-organization and intelligence.

Insect colonies distribute resources and tasks to each unit in order to collectively solve difficult problems instead of using any centralized control. The behaviour comes from the local interactions with no global knowledge and it uses a simple set of rules [5]. These rules provide robustness to change, keeping their effectiveness in extremely diverse environment or recourse distribution over a broad range of tasks [5].

The usage of Global Positioning System (GPS) in the formations of autonomous airships has been discussed in [6]. Experiments were carried out using two blimps, both equipped with multi carrier-phase GPS (CPGPS) receivers allowing the establishment of attitude and location of the craft. The experiment identified formations with attitude errors. The errors were in the range of less than five degrees with further positional errors of less than a foot. Formation control is one approach to comprehend cooperative coordination proposed in [7]. Multi-UAV formation flight combines the research of both UAV and coordination, so it has increase interest from both unmanned system and control communities [8]. Cooperative coordination allows that a group of UAVs can follow a predefined trajectory for flight missions while using their on-board sensors to acquire useful information while maintaining a specified formation pattern. Because formation flights of a UAV fleet can significantly increase the universal efficiency of the entire system, it can benefit most of the applications which are handled by a single UAV.

The functionality of compound eyes in animals has been described extensively in literature. The Compound eye is excellent in motion detection [9]. Different facets of the eye are progressively stimulated when objects move across the visual field of the insect. This is call the "flicker effect", which makes the insect respond far better to moving objects 
than to unmoving ones. [1] Has shown that compound eyes like that of a moth can also show a very high sensitivity to light. This makes some insects, such as the praying mantis (archimantis latistyla), a good hunter even during the early and late hours of the day. It has also been shown that some insects such as the drone fly (eristalis tenax) can see ultraviolet as well as natural colour light [2]. This helps drone flies to forage together with the honeybees they are mimicking.

The compound eyes of most insects have many hundreds of lens facets. This provides these insects with a very wide field of view. [10] Shows that composite lens eyes of some insects having no more than 50 facets can still show excellent performance. The issue of seeing the world multiple times with different view angles is compensated by the cortex of these insects. [11] Describes a design of a composite eye for computer vision for detecting moving objects in a closed environment. They designed a small unmoving array of seven off-the-shelf cameras and show that it is able to track a moving object with this kind of system. The 3D position of the object can also be determined in the overlap areas. In [12], a compound eye is modeled using a spherical field of view, overlapping Gaussian-shaped receptive fields, a singular viewpoint, and a space-variant receptor distribution. The algorithm creates low resolution spherical images from multiple static perspectives. For representing spherical images, the 3D images are projected onto cubes because current raster graphics technology is optimised to construct planar, perspective images.

After motion detection, the most important part is to identify the moving object. Identification of an object autonomously is a complex task as most real environments have many variations which can affect the image and visibility of the scene. [13] Proposed a method of autonomous real-time vehicle detection from a medium-level. The detection of moving vehicles is vital in tactical decision making for military. The general problem of aerial vehicle detection is also made significantly more demanding due to the nonuniformity of vehicle colour, localised shape characteristics and overall dimension. To determine the relative position within an unknown terrain is a challenging task. The DGPS /AGPS receivers make a fine selection for UAV positioning main sensors, because they are able to reach accuracy up to few centimetres using carrier-phase measurements. DGPS /AGPS receivers are the only position sensors that are generally used for UAV positioning [14]. The reliability of their measurements is critical for UAV missions [15].

The deployment of multiple eyes/cameras on key location could offer a high sensitive and effective motion detection solution. Real-time multiple images can be exploited and analysed. More cameras in the right locations mean fewer blind spots. Most of the motion detection algorithms are based on comparing the frames with each other and some also compare the frames with each other with the dimensions of time to extract the moving object's speed.

This paper exploits the concept of compound eyes in a multi-UAV (i.e. multiple cameras) surveillance application. This, a swarm formation algorithm for optimal automatic positioning of multi-angle 3D visual information sources and implementations will be the contribution of this paper.

The outline of this paper is as follows: Section 2 describes the biological background related to insect compound eye and its relation to Swarm Eye. Sections 3 explains the visibility factor index concept and proposed formation algorithm for dynamically positioning multiple UAVs. A software implementation of the Swarm Eye algorithm and test results are described in Section 4. Finally, conclusions are drawn in Section 5.

\section{BIOLOGICAL BACKGROUND}

\section{A. Insect Compound Eye}

A compound eye can consist of hundreds of individual photoreceptors. The image perceived is a combination of inputs from the numerous ommatidia (individual 'eye units'), which are located on a convex surface, thus pointing in slightly different directions, see Fig. 1. Each ommatidia consists of a lens, transparent crystalline cone, light sensitive visual cells and pigment cells.

To compare with simple eyes, compound eyes possess a very large view angle and can detect fast movement and, in some cases, the polarization of light. Because the individual lenses are so small, the effects of diffraction impose a limit on the possible resolution that can be obtained. This can be countered by increasing lens size and number. To observe with a resolution comparable to our eyes, humans would require compound eyes which would reach the size of their head [16].

The compound eye is excellent at detecting motion. As an object moves across the visual field, ommatidia are progressively turned on and off. Because of the resulting 'flicker effect', insects respond far better to moving objects than stationary ones. Honeybees, for example, will visit windblown flowers more readily than still ones.

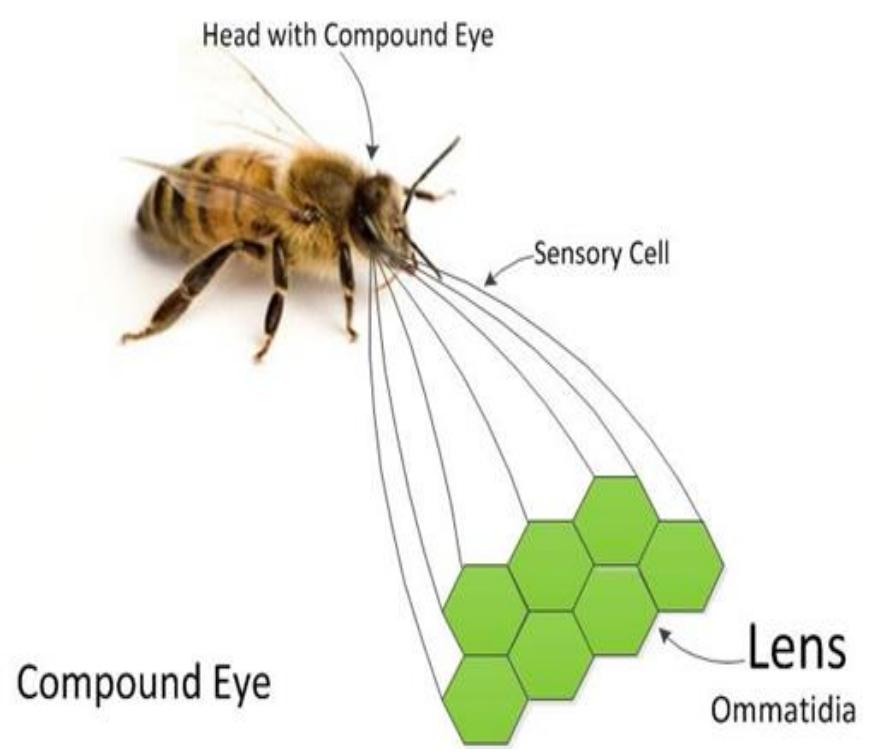

Fig. 1. Basic Structure of Compund Eyes. 


\section{B. Swarm Eye}

The Swarm Eye philosophy follows the idea that a set of individuals can form a very powerful system when joined and self-organized in a swarm. The individuals in a swarm can communicate and interact with each other directly or indirectly. The Swarm Eye System consists of several independently running units that can communicate in any specified way. The units can also take images while navigation through a simulated environment. The images can be combined in any fashion for generating e.g. high quality panoramic images, 3D image generation, and automatic position determination in case of GPS malfunctions in a tactical situation or rapid movement detection in a scene. The intelligent combination of multiple units gathering images and processing them at the same time leads to techniques that can make use of the full power of parallel images processing.

Swarm Eye follows the idea of using method motivated by nature to shape swarms. Swarms are structures caused by the emergent behaviour of individuals. Natural swarms prove to be very effective while being robust against variations in the environment or even loss of individuals from the swarm. Swarms follow rules that can lead to extremely complex behaviour [17]. The rules are often surprisingly simple and the components of the swarm are very basic [17]. However, the emergent behaviour leads to very powerful and effective results. The Swarm Eye system is a distributed system therefore the loss of a few individuals may compromise the performance, but the system will be in working order.

The Swarm Eye is a tactical decision support system that mainly uses optical information. It can be airborne or land based. The swarm consists of a scalable number of rather simple units that collaborate in a self-organized way. The Swarm Eye concept is designed for medium to large scale swarms. The swarm unit numbers can range from a couple to several hundred units. The Swarm Eye is designed to operate in the field, where several sources of information are needed to make well informed tactical decisions.

In nature, the compound eye is excellent at detecting motion. The concepts that can be adapted to Swarm Eye applications are:

- To have many eyes /camera

- Observe the object from many different angles

In Swarm Eye, the camera locations can be in different places, whilst in compound eye each ommatidia is fixed in pace next to each other. The eye units are the basic components in both the compound eyes and Swarm Eye.

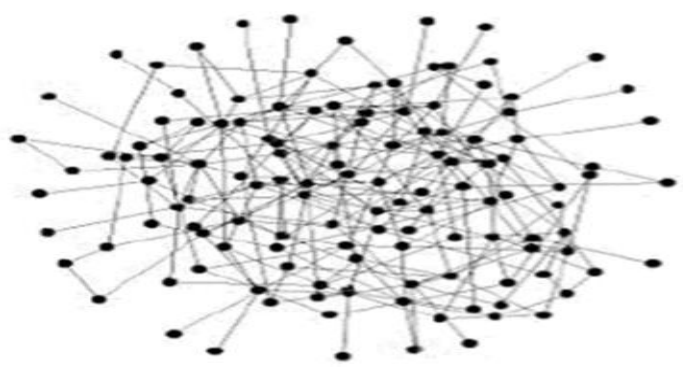

Fig. 2. Decentralized System.

\section{DisTRIBUTED SURVEILLANCE ALGORITHM}

\section{A. Decentralized Communication}

In a centralised communication approach, every unit in the swarm communicates with one centralised hub and the central hub sends commands/messages to all other units. The drawback of this approach is that if the centralised hub fails then the whole system will eventually fail.

There are different schemes to implement a decentralised communication model. In general, decentralised communication requires each unit to broadcast the message to every unit, see Fig 2. These systems are more robust as they do not rely on any central hub of the system. They are also fault tolerant as the failure of a communicating unit does not adversely affect the functionality of the whole system. In the development of Swarm Eye, the adopted communication approach is distributed decentralized. This is due to the requirements of autonomous operations and fault tolerance.

\section{B. Swarm Eye Formation}

The identification and implementation of the proper formation of swarms is a vital element for efficient swarm application. These formations typically occur during the various phases of the swarm lifecycle.

The initial formation refers to the state of the swarm upon its creation; however this initial formation can be changed accordingly based on the swarm deriving parameters such as visibility. This adoption of a new formation overcomes any drawbacks associated with the static formation limitations. Within the context of aerial surveillance this dynamic formation is a desirable behaviour as it aids in overcoming obstacle avoidance and any static restrictions. It also allows for maximum coverage of any area of surveillance from the sky.

The formation is derived by an algorithm that is applied on the swarm members to achieve a particular orientation of these members within the swarm. To aid this process, this paper proposes a visibility factor index driven algorithm to apply to a suitable swarm formation. This algorithm comprises of several steps as depicted in Fig. 3.

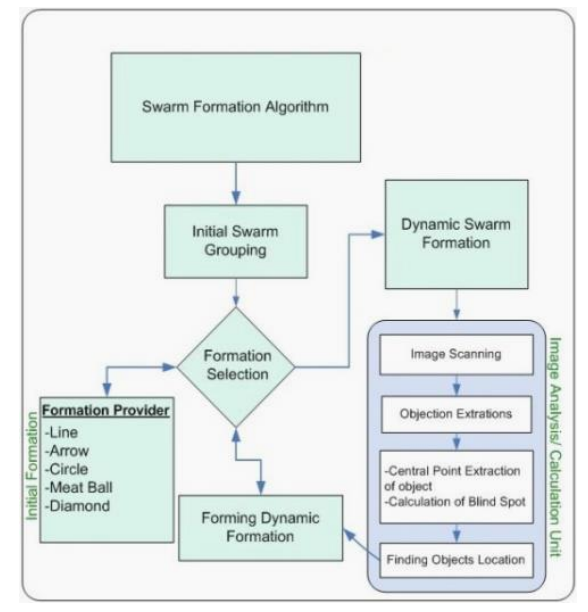

Fig. 3. Architecture of a Decentralized Swarm Eye Flying Formation Algorithm. 
These steps aid in achieving a suitable formation for the swarm. The algorithm aims to apply a suitable formation to the swarm so that maximum visibility can be achieved over an area of surveillance.

\section{Visibility Factor Index}

The visibility factor will be decided depending on how much of the required object is visible to the individual eye. Each eye is assigned a rank. The eye that will cover more area of the required object that eye will have higher Visibility Factor Index (VFI), i.e. rank. VFI ranking is shown in more detailed in Fig 4.

Fig. 4 shows four swarm units; each unit with its own point of view. The Unit 1 can see more of the required object (the car behind the tree) - therefore in the ranking table it is ranked higher than the others. The VFI ranking is dependent on the visibility, therefore, higher visibility yields higher VFI values and vice versa. The VFI aids the swarm system to establish swarm units with higher visibility at their present location. On the other hand, VFI allows for selection of low VFI swarm units for relocation in order to improve their VFI. The swarm formation algorithm also utilises low VFI swarm units as candidates for location transformation and swarm formation.

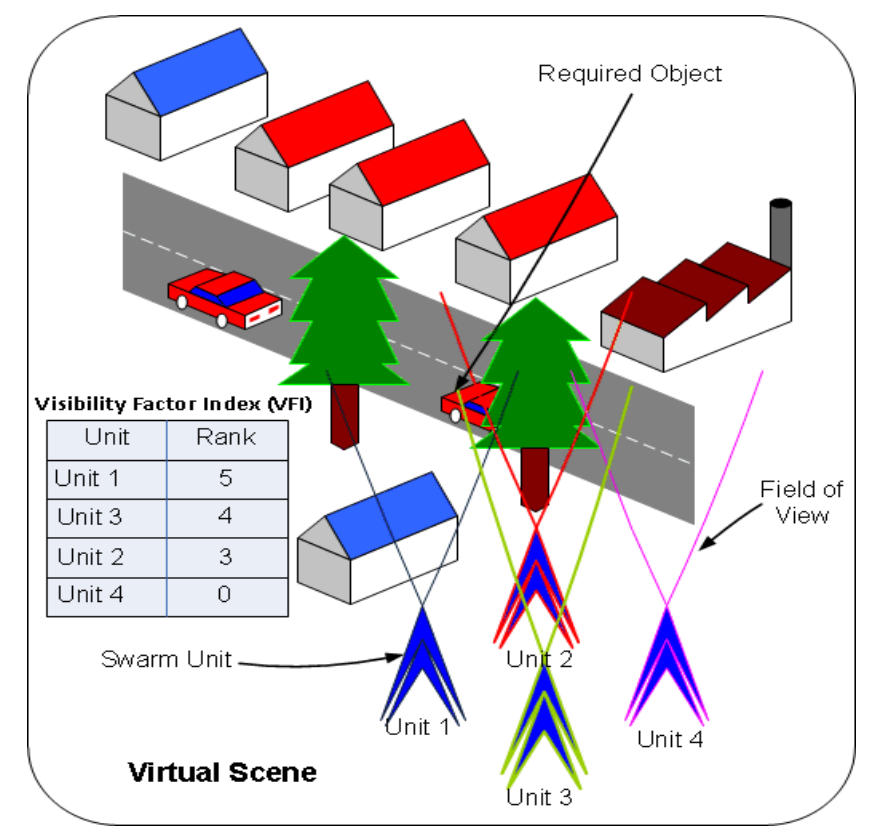

Fig. 4. Virtual Scene Demonstration of Visibility Index Execution.

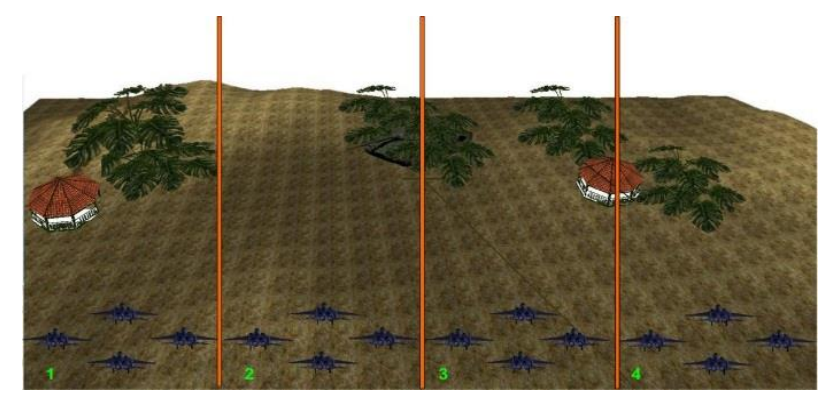

Fig. 5. Example of Full Swarm Dividing into Four Small Sub-Groups with Four UAV Each.

\section{Formations}

The swarm formation can be divided into 1) Forming Initial Formation and 2) Forming Dynamic Formations. The initial formation refers to the formation which will be adopted by the swarm at the start up. As soon as the unit will be airborne and get registered into the swarm they will form a formation to fly, which could be arrow, diamond, semi-circle, circle formations etc. This initial formation can be decided based on a few factors, for example if a swarm unit intents to save fuel then they will adopt the arrow formation [18], [19], and [20]. In the case of poor visibility, the formation will switch into a dynamic formation offering maximum visibility. The dynamic and initial formation algorithms are explained later in this paper.

The dynamic formation will be adopted by the swarm if the required objects are not visible due to blind spots in the current formation. The proposed formation algorithm presented in this section aims to derive a suitable formation based on the Visibility Factor Index. The objective of the algorithm is to apply a suitable formation to the swarm so that maximum collaborated visibility is achieved. The algorithm divides a swarm into several sub-swarms applying suitable formations at both, the sub-swarm and the overall swarm level, see Fig. 5. This approach allows for individual subswarms within a main-swarm to have their own formation. With the main swarm divided into multiple individual subswarms each can have its own unique formation. This allows for a mix of formations within a main swarm that can be changed accordingly as desired.

The algorithms attempt to create sub-swarms of similar number of units by applying a square root function to the number of units in the swarm. For example, if the total numbers of units are 16 then applying $\sqrt{16}$ will yield 4 . The formation will make 4 groups, each group containing 4 units (in other words $4 \times 4=16$ total swarm units) as shown in the above Figure 5. In the case of any leftover unit, these units will join the nearest group. Each group can be used for different purposes as well as share the same task and each group can perform a certain job or task. The square root function approach has been selected due to the simplicity of implementation and it also allows for creation of equal number of groups with similar number of units. A fair distribution of tasks to individual units is also guaranteed by this approach. Various other approaches such as clustering can also be applied as a replacement to this approach. However the intent of this function is allocate units to swarm and if required it can be easily replaced by more efficient similar function.

Once all the units are assigned a group, each group will search its own allocated task. If the group faces an object/terrain with blind spots it will scan all the eyes visual index numbers. The eye which has the best ranked visual index of the required object will scan the required object's image and calculate the centre of the object $(\mathrm{ObjC})$ from its own view. After locating the centre point of the object it will calculate the distance ( $\mathrm{dBS}$ ) between the ObjC and blind spot (BS) and after calculating this distance (dBS) the system will scan the visual index again. The eye which has the lowest 
visual index and is closest to the destination point will shift its position to a surveillance point which will allow it to cover the previously hidden area of the object. This process will iterate itself until the blind spot of the required object is optimally covered. The overview of the algorithm is shown in the Fig. 6.

The algorithm has been partially implemented and is not fully optimised at this stage. Specifically there may be a case of repeated iterations to cater for the dynamicity of the scene. These iterations might need further optimization but this issue has been left for future work. However, it allows for the exploration of this novel approach within tactical decision making scenarios. Moreover, there are yet features which have to be incorporated into the algorithm, such as obstacle avoidance, keeping the formation, collision avoidance, etc. The swarm formations are designed to have units from 16 to 250; however, the general design idea of Swam Eye is not limited to any number of individuals.

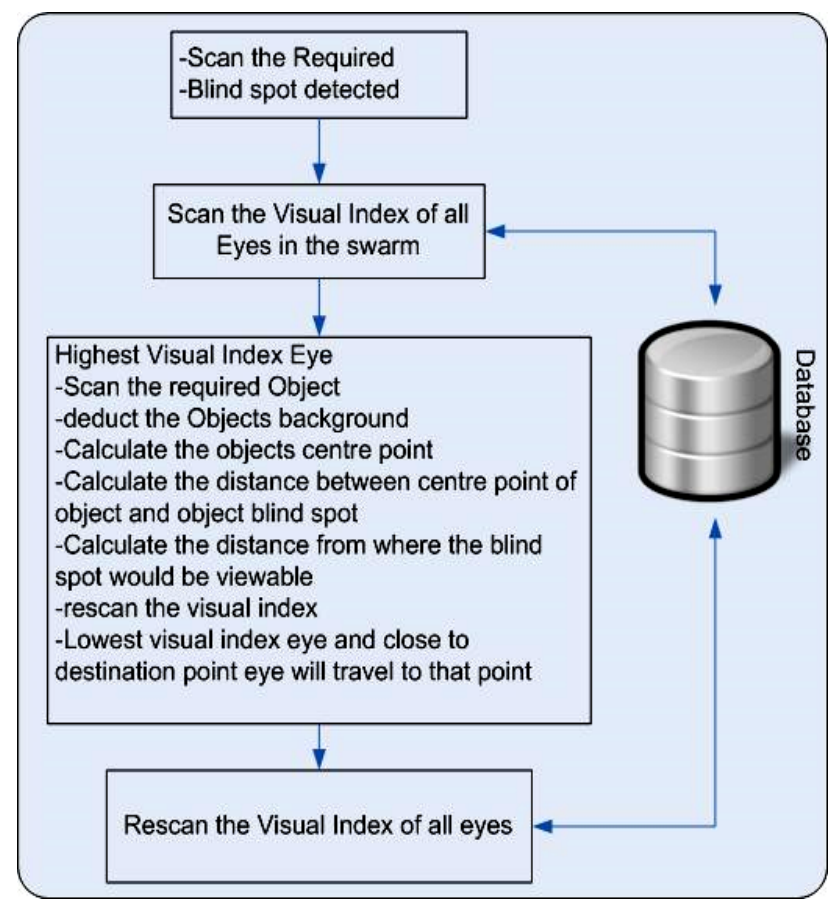

Fig. 6. Decentralized Dynamic Formation Algorithm.

\section{IMPLEMENTATION AND RESULTS}

\section{A. Prototype Software}

The Swarm Eye software prototype comes with a simulation environment for testing various application setups. A safe simulation environment has been chosen to prepare for real-world tests with UAVs. The basic principle is to provide a set of autonomous eye units which can analyse a tactical scene in parallel, running efficient image analysis in a distributed manner and, thus, providing richer information to the tactical decision maker in the field. The first steps into this direction have been made by providing a parallel communication platform. The software also delivers a parallel scene simulation and analysis. After that all the essential steps are made to start with the first distributed image analysis processes. The software system consists of

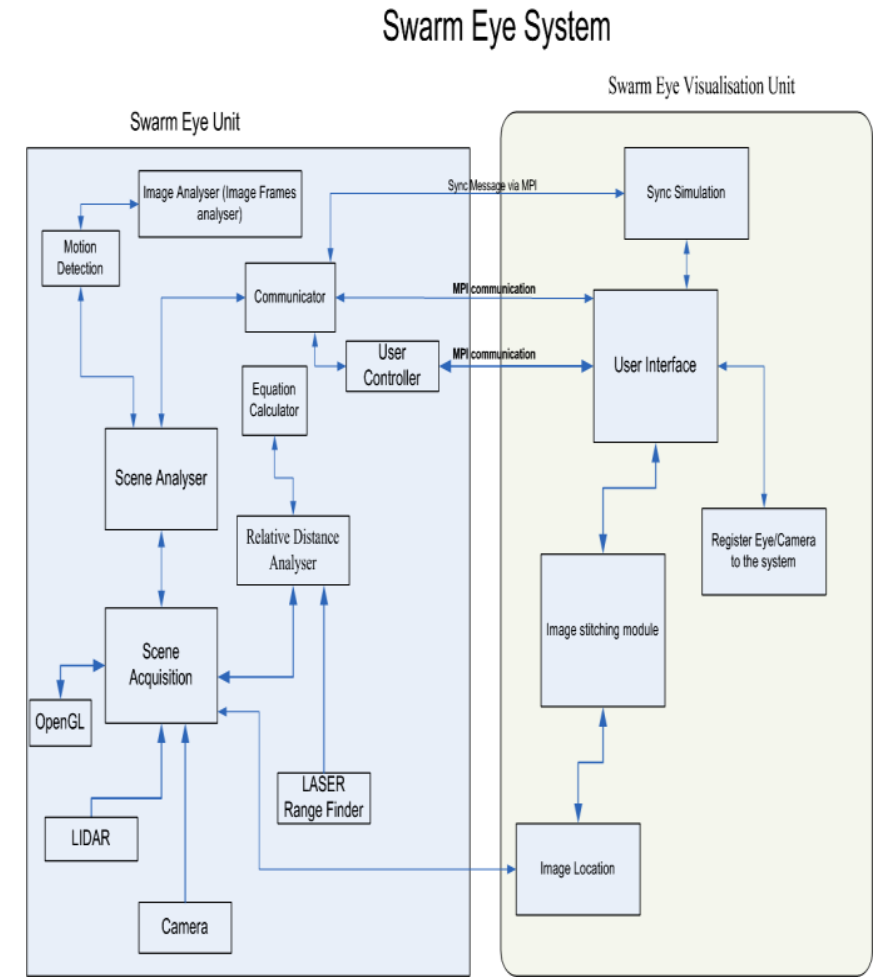

Fig. 7. Swarm Eye Software Structure.

- warm environment

- Swarm Eye units

- Parallel image processing capabilities

- Parallel communication

- User interface

Fig. 7 shows the structural layout of the Swarm Eye software. Although the current implementation consists of several individual modules, the system can be divided into two major parts: the Swarm Eye Units and the Swarm Eye User Interface (Visualisation Unit).

The visualization unit is a separate object in the Swarm Eye system. It connects to the individual Swarm Eye units via MPI (Data exchange interface). The major purpose of the visualisation unit is to link the user with the swarm. Through the registration module each eye is registered at the Visualisation Unit. This way each eye can be addressed and manipulated independently by the user. The environment is simulated in each Swarm Eye unit independently. This allows for highly efficient communication between the eye units as only small changes happening in each eye-environment simulation need to be communicated. A panoramic image generation module which uses stitching techniques is implemented in the visualisation unit. The individual images from the activated Swarm Eye units are collected by the visualisation unit and processed centrally. However, it is not necessary to have only one visualisation unit. The unit can log on to any eye in the swarm, thus avoiding a vulnerable hubspoke configuration where taking out the hub causes a failure of the whole system. 
The Swarm Eye units are the eyes/cameras of the system. They are programmed as objects so they can be copied and independently used as often as desired by the user. This way it is easy to build a swarm of many similar eye units that can interact with the environment, the user and with each other. In each Swarm Eye unit a scene analyser interacts with the scene acquisition module which interacts with the OpenGL [21] environment, a LIDAR (Light Detection And Ranging) data reader, a camera (for real-world applications), and a relative distance analyser. The motion detection module interacts with the scene analyser. Each eye has a fast communication interface (Communicator) to the visualisation unit and the other eyes. The user controller interprets direct commands from the user for e.g. activating a feature or changing the orientation or position of an eye unit. The eye unit can interpret high level commands and execute them independently. This simplifies the control of the swarm and avoids direct low level (protocol level) interaction between user and the single swarm unit.

The Swarm Eye consists of a set of autonomously performing eye units. In order to perform the task in a group, each individual needs to have a fully duplex communication protocol in real time (without significant delay). The messages between the units would be different types of data i.e. images, simple $\mathrm{x}-\mathrm{y}-\mathrm{z}$-coordinates and messages /commands. Individuals in swarms interact either directly by communication or indirectly through joint behaviour. In Swarm Eye each eye unit can communicate with each other and the visualisation unit via the quasi-standard internet peerto-peer protocol MPICH2 [22]. All processes in Swarm Eye are running in parallel and can communicate asynchronously. The communication unit also provides a platform for launching new eye units, communication between the objects and to delete the present eye/camera if needed. After each eye is released, it acts as an independent process which runs independently from the initialisation unit. This mimics the independent implementation of each eye unit on an e.g. airborne UAV.

The virtual environment simulation has been created to simulate the camera (eye). This is a 3D simulation which will allow placing a camera or eye at any desired location in the scene. A simulation is cost and time effective as compared to a real camera. There are a few simulations which have been developed and have been adopted. The simulation has been plugged into the GUI as a module and can be replaced by different simulations or by real cameras. There are few simulations that have been created from the start of the project which are all developed in $\mathrm{C}++/ \mathrm{C}$ and by using OpenGL. The $\mathrm{C}++/ \mathrm{C}$ offers to be very effective in processing consumption which is very crucial for this project.

The image rendering module is a key module in Swarm Eye. It supplies the eye units with images from the simulated environment. Significant time was spent in this work in developing an image rendering package that provides simulations that looks as realistic as possible. Until now, the image rendering module is the principle platform for experiments in Swarm Eye. All following experiments on collective image representation, position determination and movement detection have been implemented using this module. The image rendering engine used in Swarm Eye uses the MD2 file format and the OpenGL library for image rendering. During the project the image rendering module in Swarm Eye was significantly modified and improved from a very basic triangulation viewer to a sophisticated $3 \mathrm{D}$ rendering engine.

\section{B. Collective Image}

In Swarm Eye, the problem of generating panoramic images has been solved by image stitching. Image stitching is the process of combining several images together to form one single new image showing the content of the individual images in one single picture. The technology of image stitching is rather advanced today [12], [23]. Swarm Eye makes explicit use of these advanced technologies and incorporated them into the system. Fig. 8 shows the general structure of the panoramic image generation process.

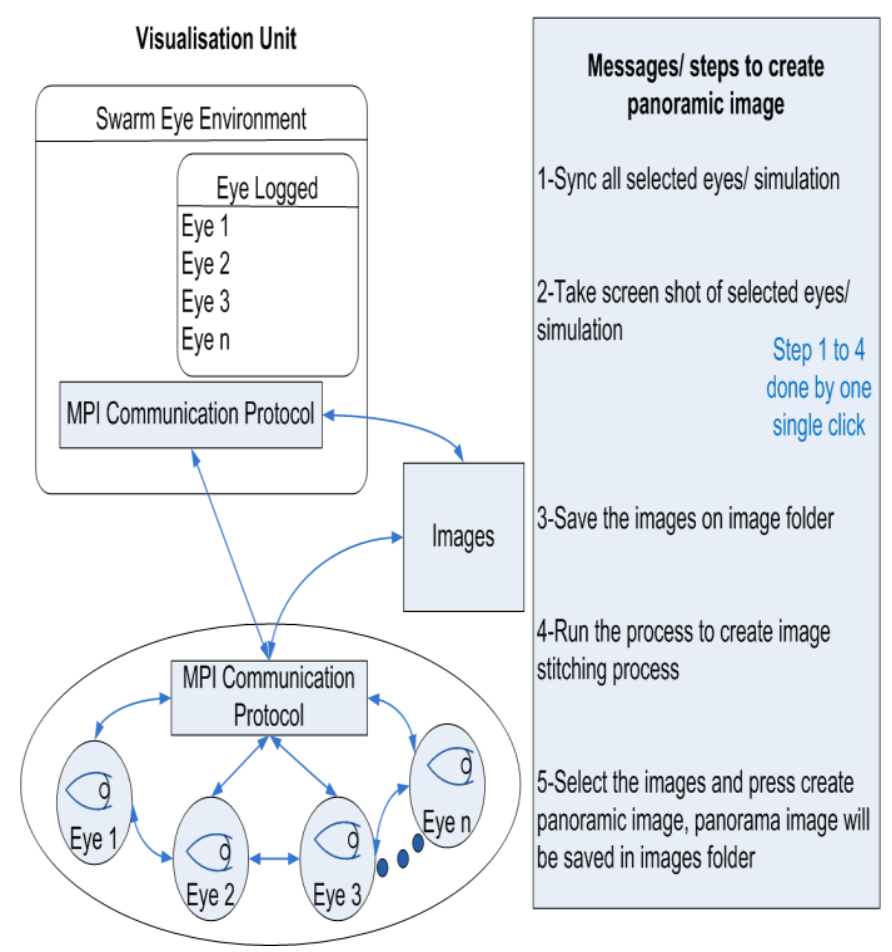

Fig. 8. Panoramic View Generation in Swarm Eye.

The eye units contributing to the panoramic image generation are first selected in the visualisation unit by the user. The scene is synchronised via MPICH2 messages so that every eye has the same time frame. In real-world scenarios the synchronisation procedure is, of course, not necessary. Each eye takes a snapshot of the scene and sends it to the Visualisation Unit. In order to perform image stitching in Swarm Eye, the individual views of the eye units need to be synced and offer overlapping coverage of the scene. In practice any orientation of the eyes can be used for this purpose as long as there is a certain amount of overlap between the individual images. An overlap of ca $20 \%$ between each image is generally recommended to allow the stitching software to identify unique markers within the overlap area to determine stitching points. The marking procedure is performed by the Scale-Invariant Feature Transform algorithm 
SIFT [24]. The RANSAC (RANdom SAmple Consensus) [25] algorithm is used to compare similarities between the point sets identified by SIFT in the individual overlaps. The images are adjusted and transformed so that similar point clusters overlap with the highest probability. The adjusted images are then rendered and combined into one single image. In Swarm Eye, the images are collected and passed to the image stitching software to produce panoramic view [13], [26].

In Fig 9 the views of three eye units are displayed showing a landscape. One can see that each eye is pointing in a slightly different direction covering the major area of a scene.
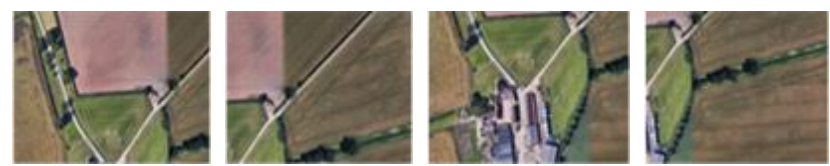

Fig. 9. View of Different Eye units.

Fig 10 shows the compiled panoramic view. After activation of image stitching, this image can be seen. All four images are sent by the eye unit to a shared common port. From there the stitching software automatically collects the images and joins them to a single panoramic image. The image is displayed automatically by the Swarm Eye to a user on the ground who logs into this viewing unit.

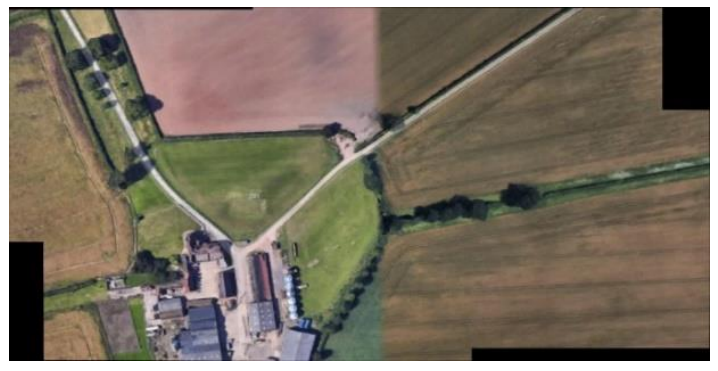

Fig. 10. Panoramic View Generation in Swarm Eye

\section{Swarm Formation}

The formation algorithm has two parts in the software development. The first part is to form the initial formation and the second part is to form a dynamic formation when a blind spot is being detected or the required object is not visible anymore. This approach can be based on particle swarm optimisation [27]. The visibility factors, object of interest depth perception, assisted technologies such as LIDAR usage also contribute towards format adjustments, see Fig. 11.

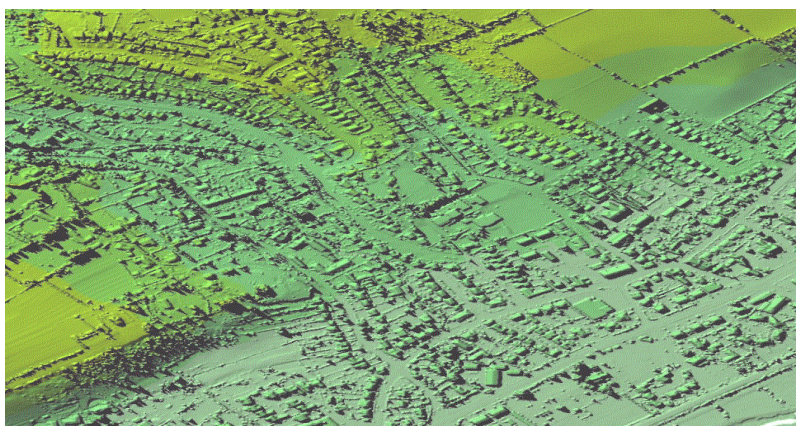

Fig. 11. Aerial LiDAR View of a Landscape.

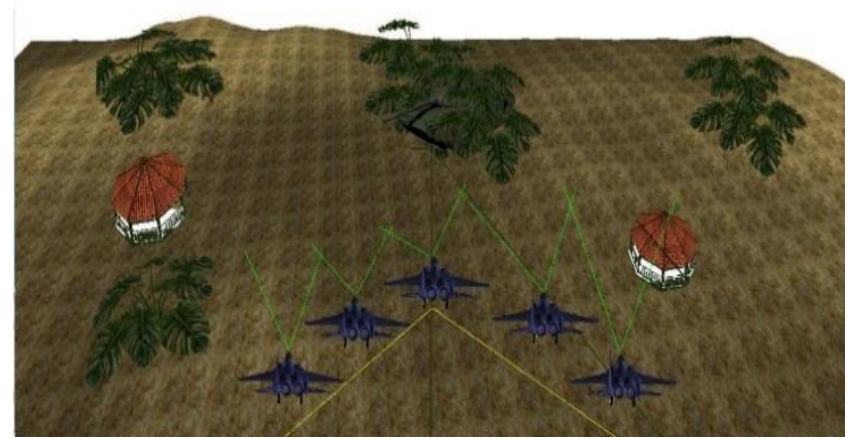

Fig. 12. Arrow Formation of Swarm units.

The selected formation is applied at the initial stage of the swarm lifecycle. If during the simulation a blind spot is encountered by a swarm unit then this event triggers a dynamic reformation of the swarm. Individual swarm units have a limited area of observation. However, when multiple swarm units are placed in an appropriate formation, this yields a larger area of coverage. To maintain the optimum collaborated coverage of the area of surveillance, the individual swarm units maintain a sight distance between each other. Fig 12 presents this scenario where five swarm units are arranged in an arrow formation with the whole swarm coverage area. This feature allows for wide range panoramic views of the scene of interest to be generated.

However, the generation of $3 \mathrm{D}$ images from 2D captured images requires suitable multi-angled $2 \mathrm{D}$ images. This is where dynamic formation plays a vital role upon identifying a suitable object of interest. The formation will consider the number of units in the swarm, the requirement to cover the object of interest from various angles and then apply a suitable formation to the swarm. This scenario is presented in the Fig 13, where the hidden tank (in the middle of the scene) acts as the object of interest and has been covered by the various units in the swarms from different angles.

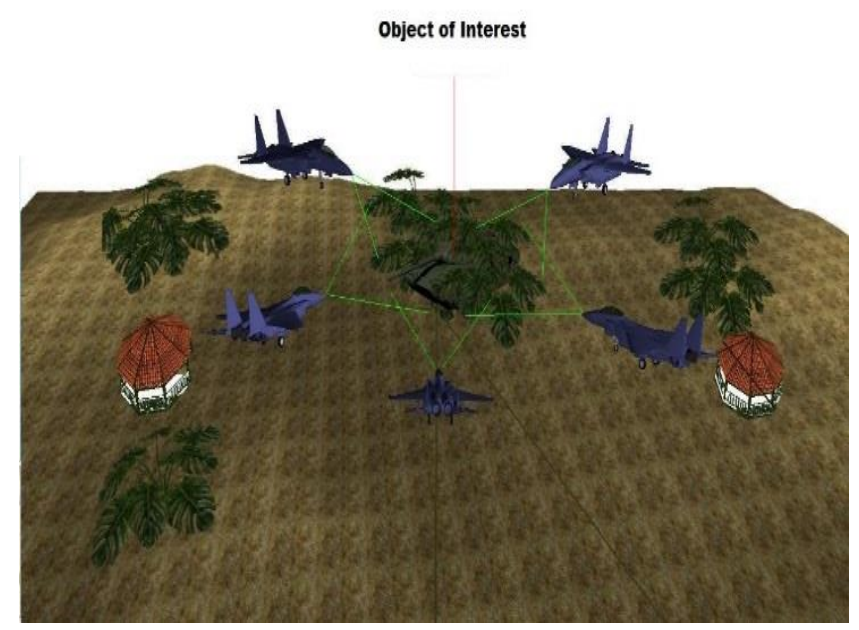

Fig. 13. Demonstration of Dynamic Formation. 
These dynamic formations are essentially objective driven. The objective may vary from multi-angle surveillance of a specific object of interest to acquisition of sufficient multi angle data to construct a 3D representation of a tactical scene.

The dynamic transformation is governed by the following selected steps of the algorithm

Step 1: The required object within the tactical scene is scanned by each individual eye. This will generate the visual index of the individual eyes.

Step 2: The Unit with the highest visual index and closest to the required object will subtract the background from the object and then detect the centre point of the visible required object.

Step 3: The centre point of the required visible object is calculated and the distance from the centre point to a blind spot of the object is established.

Step 4: The calculations above will allow for the selection of swarm units for repositioning in order to increase their visual index factor and the overall collaborated visual coverage of the area.

These steps are summarised in the block diagram shown Fig 14. They have been implemented as communication between various classes within the system.

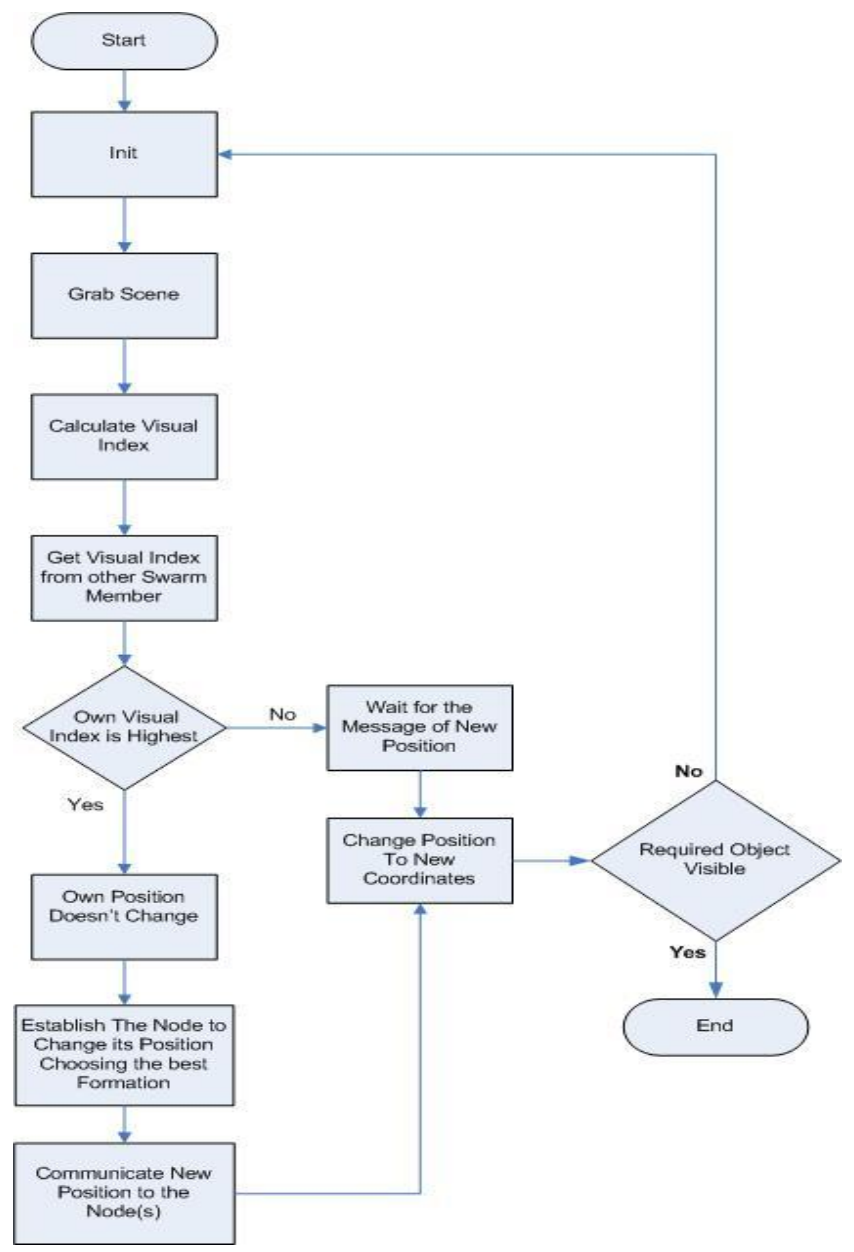

Fig. 14. Block Diagram of Dynamic Formation Algorithm.

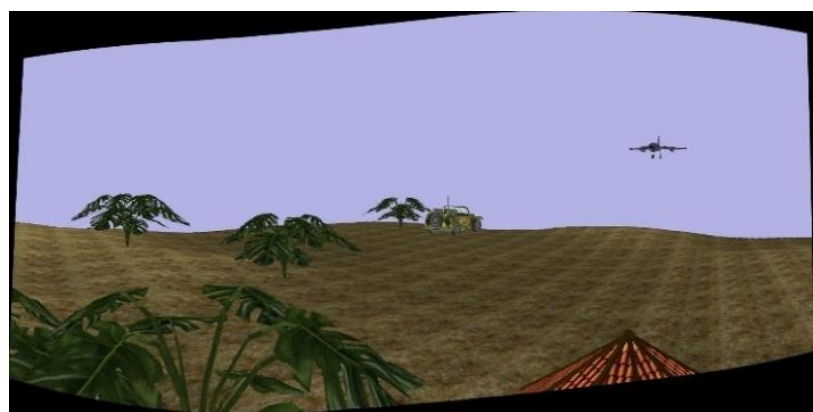

Fig. 15. Panoramic View of Swarm Curve Formation.

A swarm unit dynamically operates in a particular formation. The position and orientation of an individual swarm member within a formation affects its individual view and the overall stitched panorama. Different swarm formation coverage of a particular area of interest may yield a different type of panoramic view. This section presents some interesting panoramas created by stitching individual views of Swarm Eye units in different formations.

The panorama in Fig 15 is created by stitching individual views of swarm units in a curve formation. This panoramic image is based on individual views of 15 swarm units. The formation and orientation of the individual swarm is presented in Fig 16.

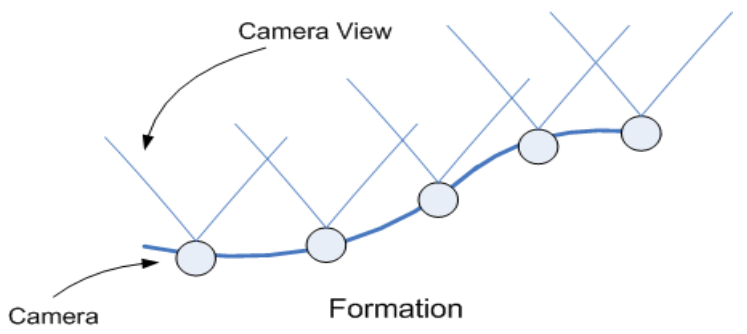

Fig. 16. Orientation of Swarm units in Curve Formation.

The collaborative viewing capability of the swarm allows for coverage of wide areas for an aerial surveillance perspective. A downward camera orientation within an arrow formation can be used to generate the collaborated view of an even wider area. An example of this case is shown in Fig 17. The panoramic image was generated by the swarm in an arrow formation shown in Fig 18 with a downward camera orientation towards the ground. This picture provides the vital information of the ground beneath the aircrafts.

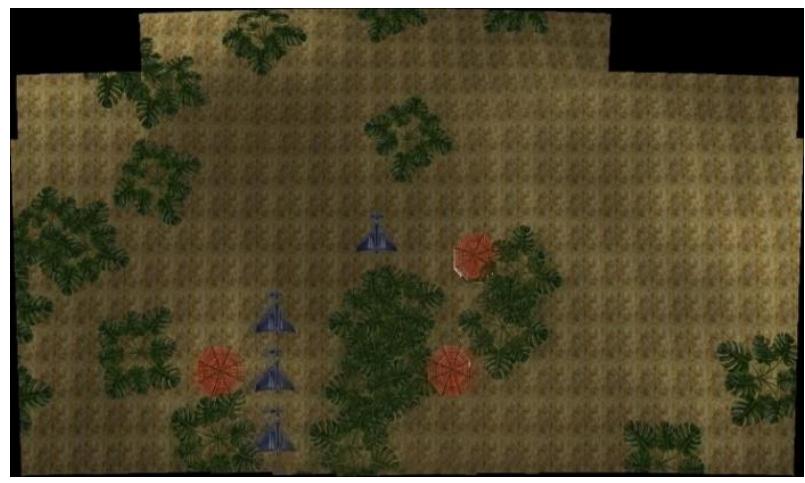

Fig. 17. Panoramic View of Swarm Arrow Formation. 


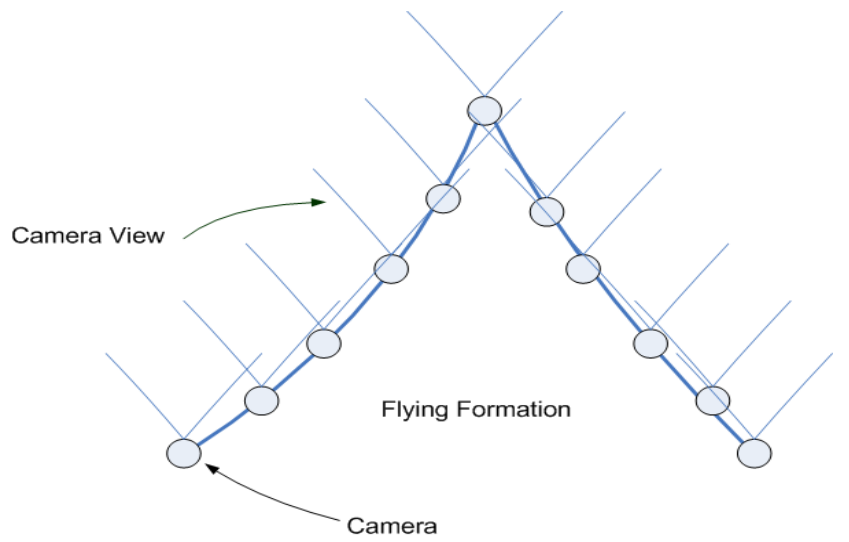

Fig. 18. Orientation of Swarm Units in Arrow Formation.

Several types of UAV can hover remaining still in the air allowing them to form formations that are usually not possible with wing based UAV. For instance helicopters can rotate in the air and this allows them to capture $360^{\circ}$ degree coverage of the area of surveillance.

Furthermore, the flying capability allows them to form a circular formation. It is also possible to change the camera orientation within a circular formation to focus towards the centre of the circle. This allows for circular angular coverage of an area of surveillance and stitching individual views can generate spherical panoramas. The semi-circle formation shown in Fig. 19 allows for multi-angle coverage of the surveillance area. A full circle formation can also aid in acquisition of 3D information about the object of interest within a scene, see Fig. 20. The quality of the generated panorama is dependent on several factors such as overlap, view orientation and the algorithm used to stitch images together. In certain instances a particular algorithm may fail to generate a panorama if any of the required parameters of the algorithm are not met.

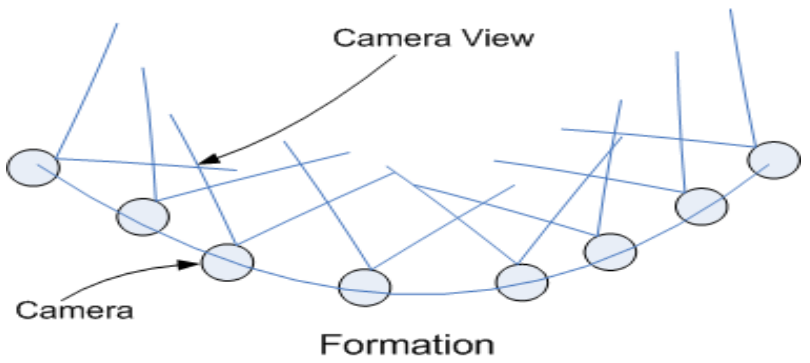

Fig. 19. Orientation of Swarm Units in Arrow Formation.

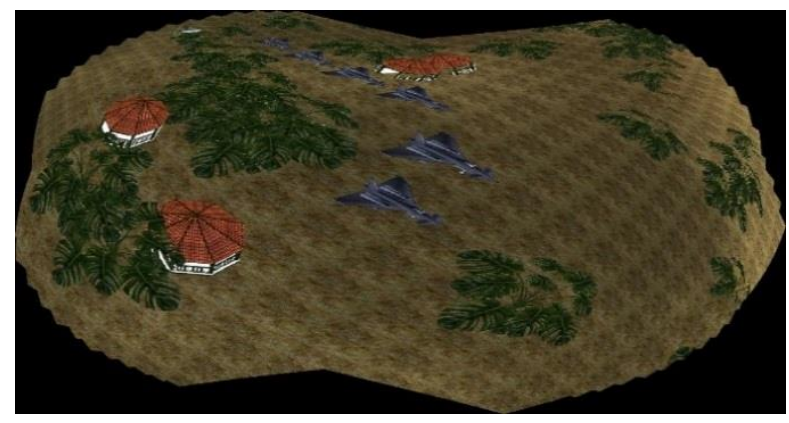

Fig. 20. Panoramic View of Circular Formation.

\section{CONCLUSIONS}

Distributed ground teams or swarms of UAVs can provide different and dynamic views of tactical scenes. Swarms are sets of interconnected units that can be arranged and coordinated in any flexible way to execute a specific task in a distributed manner. Swarm Eye provides a simulation environment to explore the potential of swarms and its merger with various advanced technologies to aid in surveillance and reconnaissance missions.

The identification and implementation of the proper formation of a swarm is a vital element for efficient swarm applications. The formation is derived by a suitable algorithm that is applied on the swarm members to achieve a particular orientation of these members within the swarm. The initial formation refers to the formation which will be adopted by the swarm at the start up. As soon as the unit will be airborne and get registered into the swarm they will form a formation to fly. The dynamic formation will be adopted by the swarm if the required objects are not visible due to the blind spots in the current formation.

In this paper, a formation algorithm has been proposed which demonstrates that blind spots can be minimised to get a better view of the target object or terrain. This formation algorithm aims to derive a suitable formation based on a visibility factor index. This index refers to a quantifiable unit that can measure visibility with regards to a particular object of interest within an area of surveillance. A higher visibility of the object of interest yields a higher visibility factor index. This quantified visibility unit is then used in an algorithm to apply a suitable formation to the swarm units.

The objective of the algorithm is to apply a suitable formation to the swarm so that maximum collaborated visibility is achieved. The algorithm divides a swarm into several sub-swarms applying suitable formations at both the sub-swarm and the overall swarm level. This approach allows for individual sub-swarms within a main-swarm to have their own formation. The division of a whole swarm into subswarms is very effective; by having sub-swarms, the user can allocate different jobs to different groups and individual subswarms are easier to manage as compared to managing whole swarms with hundreds of units.

The Swarm Eye consists of a set of independently performing eye units. In order to perform the task in a group each individual needs to have a suitable communication protocol to allow for communication between the units. The messages between the units would be different types of data i.e. images, simple 3D coordinate and messages /commands. Individuals in swarms interact either directly by communication or indirectly through joint behaviour. This coordinated behaviour allows for deployment and experimentation with the swarm of variable sizes. The proposed simulation platform provides an extensible framework for further exploration and experimentation with the autonomous swarm operations and formations with regards to tactical decision making. The self-formation capability using visual factor index allows for autonomous swarm formation and aids surveillance with reference to a specific object of interest. 


\section{FUTURE WORKS}

The article has presented a novel approach for the swarm formation based on VFI. This approach allows dynamic swarm formations with the focus of maximum coverage of an object of interest. This approach can aid in tactical decision making and allows for autonomous swarm behaviour. This research study was constrained by time and budge and further research development can be carried on in the following points.

- The initial formation algorithm has been implement ed but the dynamic algorithm implementation is to be undertaken.

- The optimised implementation of the swarm formation algorithm with the integration of other collaboration algorithms such as (collision avoidance, obstacle avoidance, target seeking and formation keeping) at present the Swarm Eye system does not fully implement these methods.

- Implementation of image analysis by swarm unit to help tracking, detecting the target and form dynamic form ation by applying the swarm formation algorithm.

- The analysis of real time constraints and time delay of the Swarm Eye formation building and processin $\mathrm{g}$ of the visual data by the swarm units could be imple mented and tested on real units rather then simulation.

- The UAV physical manoeuvring constraints to be catere $\mathrm{d}$ by the algorithm in more detail.

The swarm formation is a considerable new topic and minute research has been performed. The area of swarm formation, coordination of multiple autonomous flying vehicle offers diverse edges of Swarm and still need further exploring and development.

The Swarm Eye project is open to many applications; this project provides the foundation of the Swarm Eye concept and could also benefit different industries.

\section{ACKNOWLEDGMENT}

This work is part of a research degree program at Cranfield University. The authors would like to thank an industrial sponsor, Lockheed Martin Corporation of this project for financial and technical support during the course of this study.

\section{REFERENCES}

[1] G. Struwe, "Spectral Sensitivity of the Compound Eye in a Moth. Intra and Extra Cellular Recordingds," Acta Physiologica Scandinavica, vol. 87, no. 1, pp. 63-68, 2008

[2] L. Bishop, "An ultraviolet photoreceptor on a Dipteran compound eye," J.comp. Physiol, vol. 91, pp. 267-275, 1974.

[3] "Online," [Online]. http://news.bbc.co.uk/1/hi/world/americas/4808342.stm..

Available:

[4] H. Xu, H. Guan, A. Liang and X. Yan, "A Multi-Robot Pattern Formation Algorithm Based on Distribution Swarm Intelligence," in Second International Conference on Computer Engineering and Applications, 2010.
[5] M. J. Mataric, "Designing emergent behaviours: from local interactions to collective intelligence," in Proceedings of the second international conference on From anomals to animats 2: simulation of adaptive behaviour, Cambridge, MA, USA, 1993.

[6] E. A. Oslen, C. W. Park and J. P. How, "3D Formation Flight Using Differential Carrier-phase GPS Sensors," in Institution of Navigation GPS Meeting, Nashville, TN, 1998.

[7] A. Ryan, M. Zennaro, A. Howell, R. Sengupta and J. K. Hedrick, "An Overview of Emerging Results in Cooprative UAV Control," in Proceeding of the 43rd IEEE Conference on Decision and Control, 2004.

[8] P. K. Wang and F. Hadaegh, "Coordination and control of multiple micro spacecraft moving in formation," The Journal of the Astronautical Sciences, vol. 44, no. 3, pp. 315-355, 1996.

[9] S. Miller, All Kind of Eyes, Marshall Cavendish, 2007.

[10] E. Buschbeck, B. Ehmer and R. Hoy, "Chunk versus Point Sampling: Visual Imaging in a Small Insect," Science, vol. 286, no. 5442, pp. 11781180, 1999.

[11] R. Koshy, R. Munasinghe and A. Davari, "A Design of a Composite Eye for Computer Vision," System Theory, pp. 284-288, 2008.

[12] T. Neumann, "Modeling Insect Compound Eyes: Space-Variant Spheric Vision," Lecture Notes in Computer Science, pp. 360-367, 2002.

[13] T. P. Breckon, S. E. Barnes, M. L. Eicher and K. Wahren, "Autonomous Real-time Vehicle Detection from a Medium-Level UAV," in in Proc 24th International Unmanned Air Vehicle Systems, pp. 29.1-29.9, 2009.

[14] J. Tisdale, Z. Kim and J. Hedrick, "Autonomous UAV path planning and estimation," IEEE Robotics and Automation Magazine, vol. 16, no. 2, p. $35,2009$.

[15] G. Heredia, F. Caballero and I. Maza, "Multi-UAV Cooperative Fault Detection Employing Vision-Based Relative Position Estimation," in 17th World Congress, The International Federation of Automotic Control, Seoul, 2008.

[16] R. Fernald, "Casting a genetic light on the evolution of eyes," Science, vol. 313, no. 5795, pp. 1914-1918, 2006.

[17] M. J. Krieger and J. B. Billeter, "The call of duty: Self-organised tast allocation in a population of upto twelve mobile robots," Robotics and Autonomous Systems, pp. 65-84, 2000.

[18] B. R. Cobleigh and J. L. Hansen, "Induced Moment Effects of Formation Flight Using Two F/A18 Aircraft," in NASA Dryden Flight Research Centre, California.

[19] P. Binetti, K. B. Ariyur, F. Bernelli and M. Kristic, "Formation Flight Optimisation Using Extremum Seeking Feedback," Journal of Guidance Control and Dynamics, vol. 6, no. 1, 2003.

[20] A. Gopalarathnam, Aerodynamic Benefit of Aircraft Formation Flight, Encyclopaedia of Aerospace Engineering, 2010.

[21] D. Shreiner, M. Woo, J. Neider and T. Davis, OpenGL(R) Programming Guide: Version 2, 5th Edition, Addison-Wesley Professionals, 2005.

[22] P. Pacheco, Parallel Programming with MPI, Morgan Kaufmann, 1988.

[23] P. Burelli, L. Di Gaspero, A. Ermetici and R. Ranon, "Virtual Camera Composition with Particle Swarm Optimisation," Lecture Notes in Computer Science, vol. 5166, pp. 130-141, 2008.

[24] Q. Zhang and H. Li, "MOEA/D: A Multi-objective Evolutionary Algorithm Based on Decomposition," Evolotionary Computation, IEEE Transactions, vol. 11, no. 6, pp. pp. 712-731, 2008.

[25] R. Roy and J. Mehnen, "Technology Transfer In Studies in Computational Intelligence," Evolutionary Computation in Practice, vol. 88, pp. pp. 263-281, 2008.

[26] J. Sokalski, T. P. Breckon and I. Cowling, "Automatic Salient Object Detection in UAV Imagery," in in 25th Bristol International UAV System.

[27] M. Duong, P. Cong, H. Quach, T. H. Dinh and Q. Ha, "Enhanced Discrete Particle Swarm Optimization Path," Automation in Construction, vol. 81, pp. pp 25-33, 2017. 